\title{
A specific polymerase chain reaction method to identify Stenotrophomonas maltophilia
}

\author{
Stephanie Wagner Gallo', Patrícia Locosque Ramos², \\ Carlos Alexandre Sanchez Ferreira', Sílvia Dias de Oliveira ${ }^{1 /+}$
}

\author{
${ }^{1}$ Laboratório de Imunologia e Microbiologia, Departamento de Biologia Celular e Molecular, \\ Faculdade de Biociências, Pontifícia Universidade Católica do Rio Grande do Sul, Porto Alegre, RS, Brasil \\ ${ }^{2}$ Departamento de Biofísica, Universidade Federal de São Paulo, São Paulo, SP, Brasil
}

Stenotrophomonas maltophilia is a multidrug-resistant nosocomial pathogen that is difficult to identify unequivocally using current methods. Accordingly, because the presence of this microorganism in a patient may directly determine the antimicrobial treatment, conventional polymerase chain reaction (PCR) and real-time PCR assays targeting $23 S \mathrm{~S}$ RNA were developed for the specific identification of S. maltophilia. The PCR protocol showed high specificity when tested against other species of Stenotrophomonas, non-fermentative Gram-negative bacilli and 100 clinical isolates of S. maltophilia previously identified using the Vitek system.

Key words: Stenotrophomonas maltophilia - identification - PCR

Stenotrophomonas maltophilia is an important emerging opportunistic nosocomial pathogen that is found in different environmental sources and it is commonly described as an infecting agent in immunocompromised, oncologic and cystic fibrosis patients (Davies $\&$ Rubin 2007, Looney et al. 2009). The treatment of $S$. maltophilia infection is difficult due to its intrinsic resistance to important antimicrobial agents and mobile genetic elements, such as transposons and plasmids, which are frequently present and carry determinants of resistance inserted in integrons (Chang et al. 2004, Nicodemo \& Paez 2007, Liaw et al. 2010).

S. maltophilia is usually isolated using a selective medium with the addition of imipenem and other antimicrobial agents and identified by biochemical methods (Foster et al. 2008a, Adjidé et al. 2010). However, the biochemical tests used to identify $S$. maltophilia, including such commercial systems as Vitek-2, API-20NE and Biolog, occasionally misidentify this species as other non-fermentative Gram-negative bacilli (Zbinden et al. 2007, Pinot et al. 2011). Moreover, the current polymerase chain reaction (PCR)-based protocols for the identification of S. maltophilia have also presented limitations related to low specificity due to the significant genetic similarity between Stenotrophomonas species and other non-fermentative Gram-negative bacilli (Berg et al. 1999, Foster et al. 2008b).

To overcome this problem, we developed a specific PCR protocol for the identification of S. maltophilia based on the design of a primer pair that tar-

Financial support: CAPES

+Corresponding author: silviadias@pucrs.br

Received 14 May 2012

Accepted 18 October 2012 gets a 278 bp fragment of the 23S rRNA gene (F, 5'GCTGGATTGGTTCTAGGAAAACGC3', and R, 5'ACGCAGTCACTCCTTGCG3'). The 23S rRNA gene was chosen due to the higher variability in this region among species of the Stenotrophomonas genus compared to the 16S rRNA gene, as determined using CLUSTALW software (data not shown). The primers targeting the $23 \mathrm{~S}$ rRNA gene were designed based on the alignment of the following sequences: S. maltophilia (GenBank accession AF273255), Stenotrophomonas acidaminiphila ATCC 700916T (EU878276), Stenotrophomonas nitritireducens ATCC BAA-12T (EU878278), Stenotrophomonas rhizophila ATCC BAA-473T (EU878279), Xanthomonas axonopodis pv. citri DAR 65864 (EU878280), X. axonopodis pv. phaseoli IAC13755 (FJ828875), Xanthomonas campestris pv. begoniae HT1-14 (JQ337960), X. axonopodis pv. poinsettiicola HN-1 (GU144282), Xanthomonas arboricola pv. poinsettiicola LMG 8676 (GU144280), X. axonopodis pv. manihotis IBSBF1994 (FJ828873), X. axonopodis pv. allii IBSBF1770 (FJ828871) and X. campestris pv. campestris DAR 65808 (EU878277). The PCR mixture was composed of $100 \mathrm{ng}$ target DNA, which was extracted according to Rademaker and de Bruijn (1997), $0.2 \mathrm{mM}$ each deoxynucleoside triphosphate (Ludwig Biotecnologia, Alvorada, RS, Brazil), $2.5 \mu \mathrm{L}$ 10X PCR buffer (Invitrogen, São Paulo, SP, Brazil), $1.0 \mathrm{mM} \mathrm{MgCl}$, 0.2 U Taq DNA polymerase (Invitrogen) and $20 \mu \mathrm{M}$ each primer in a total volume of $25 \mu \mathrm{L}$. The amplifications were performed using a Veriti Thermal Cycler (Applied Biosystems, Carlsbad, CA, USA) with an initial denaturation step at $94^{\circ} \mathrm{C}$ for $5 \mathrm{~min}$, followed by 30 cycles of denaturation at $94^{\circ} \mathrm{C}$ for $45 \mathrm{sec}$, annealing at $68^{\circ} \mathrm{C}$ for $45 \mathrm{sec}$ and extension at $72^{\circ} \mathrm{C}$ for $45 \mathrm{sec}$, with a final extension at $72^{\circ} \mathrm{C}$ for $10 \mathrm{~min}$. The amplicons were analysed by electrophoresis through a $2 \%$ agarose gel. In addition, the same primer pair was evaluated in a real-time PCR (RT-PCR) assay using a Platinum SYBR Green quantitative PCR (qPCR) SuperMix-UDG (Invitrogen). Each $20 \mu \mathrm{L}$ reaction contained $100 \mathrm{ng}$ of DNA sample, $10 \mu \mathrm{L}$ of SYBR 
Green qPCR SuperMix reagent (Invitrogen) and $0.2 \mu \mathrm{L}$ of each primer. The amplifications were performed using a StepOne RT-PCR system (Applied Biosystems) with the same annealing temperature and cycle number used in the conventional PCR described above.

The following reference strains of the Stenotrophomonas species and other non-fermentative Gramnegative bacilli were used to standardise the protocol: $S$. maltophilia ATCC 13637, S. maltophilia LMG 958T, $S$. maltophilia, formerly Stenotrophomonas africana LMG 22072 (Coenye et al. 2004), S. acidaminiphila LMG 22073, S. nitritireducens LMG 22074, five Acinetobacter baumannii isolates, three Acinetobacter calcoaceticusbaumannii complex isolates, one Burkholderia cepacia, five Pseudomonas aeruginosa isolates, two Pseudomonas spp and $X$. axonopodis. Specific amplification was detected using both PCR methods only for the S. maltophilia strains, including the sample previously classified as $S$. africana, whereas no amplification was observed for all other bacterial strains tested. The detection limits of RT-PCR were determined using the threshold cycle (Ct) values obtained from tests performed with S. maltophilia ATCC 13637 and S. maltophilia, formerly S. africana LMG 22072; the Ct values were 19.58 and 15.9, respectively. All tests were performed in duplicate. The standard deviation was 0.08 for both analyses. To test the applicability of the protocol, 100 different clinical isolates of S. maltophilia, which were previously identified using the Vitek system (bioMérieux, Hazelwood, MO, USA), were evaluated and produced specific amplicons.

One amplicon obtained from $S$. maltophilia ATCC 13637 was randomly chosen and sequenced using an ABI 3130 XL Genetic Analyzer (Applied Biosystems) automated DNA sequencer. The sequence was aligned with the S. maltophilia 23S rRNA sequences deposited in GenBank (AM743169 and AF273255) using MEGA 5.10 Beta software, presenting $100 \%$ identity.

Foster et al. (2008b) and Pinot et al. (2011) reported the use of a different region of the 23S rRNA gene as a target, but their results showed cross-reaction between Stenotrophomonas and Xanthomonas species, demonstrating low PCR specificity. Additionally, Pinot et al. (2011) developed a multiplex PCR targeting smeD and ggp S to differentiate S. maltophilia and S. rhizophila; although this technique was successful, it did not exclude the cross-reaction with Xanthomonas species. In contrast, no cross-reaction was observed with $X$. axonopodis using the protocol described in this study. It should be noted that the primer pair in this study was not evaluated against other species of Xanthomonas and/or S. rhizophila. Other studies have described protocols to detect $S$. maltophilia using RT-PCR, but the cross-reaction with Xanthomonas and/or other Stenotrophomonas species was not evaluated (Wellinghausen et al. 2004, Dark et al. 2011).

Therefore, the primers designed in this study are an important alternative to specifically detect $S$. maltophilia using rapid molecular methods, which can enable the timely determination of the appropriate antimicrobial protocol for the successful treatment of infections caused by this microorganism.

\section{ACKNOWLEDGEMENTS}

To Valdir Cristovão Barth Jr, for RT-PCR analyses support, and Doroti de Oliveira Garcia, for supplying strains of the Stenotrophomonas spp.

\section{REFERENCES}

Adjidé CC, Meyer A, Weyer M, Obin O, Lamory F, Lesueur C, Trouillet L, Biendo M, Ganr O, Eb F 2010. A sensitive, specific and predictive isolation medium developed for Stenotrophomonas maltophilia study in healthcare settings. Pathol Biol 58: e1-e5.

Berg G, Roskot N, Smalla K 1999. Genotypic and phenotypic relationships between clinical and environmental isolates of Stenotrophomonas maltophilia. J Clin Microbiol 37: 3594-3600.

Chang LL, Chen HF, Chang CY, Lee TM, Wu WJ 2004. Contribution of integrons and SmeABC and SmeDEF efflux pumps to multidrug resistance in clinical isolates of Stenotrophomonas maltophilia. J Antimicrob Chemother 53: 518-521.

Coenye T, Vanlaere E, Falsen E, Vandamme P 2004. Stenotrophomonas africana Drancourt et al. 1997 is a later synonym of Stenotrophomonas maltophilia (Hugh 1981) Palleroni and Bradbury 1993. Int J Syst Evol Microbiol 54: 1235-1237.

Dark P, Dunn G, Chadwick P, Young D, Bentley A, Carlson G, Warhurst G 2011. The clinical diagnostic accuracy of rapid detection of healthcare-associated bloodstream infection in intensive care using multi-pathogen real-time polymerase chain reaction (realtime PCR) technology. BMJ Open 1: 1-7.

Davies JC, Rubin BK 2007. Emerging and unusual gram-negative infections in cystic fibrosis. Semin Respir Crit Care Med 28: 312-321.

Foster NF, Chang BJ, Riley TV 2008a. Evaluation of a modified selective differential medium for the isolation of Stenotrophomonas maltophilia. J Microbiol Methods 75: 153-155.

Foster NF, Harnett GB, Riley TV, Chang BJ 2008b. Cross-reaction of Stenotrophomonas and Xanthomonas species in a $23 \mathrm{~S}$ rRNA gene-directed PCR for detection of $S$. maltophilia. J Clin Microbiol 46: 4111-4113.

Liaw SJ, Lee YL, Hsueh PR 2010. Multidrug resistance in clinical isolates of Stenotrophomonas maltophilia: roles of integrons, efflux pumps, phosphoglucomutase (SpgM) and melanin and biofilm formation. Int J Antimicrob Agents 35: 126-130.

Looney WJ, Narita M, Muhlemann K 2009. Stenotrophomonas maltophilia: an emerging opportunist human pathogen. Lancet Infect Dis 5: 312-323.

Nicodemo AC, Paez JIG 2007. Antimicrobial therapy for Stenotrophomonas maltophilia infections. Eur J Clin Microbiol Infect Dis 26: 229-237.

Pinot C, Deredjian A, Nazaret S, Brothier E, Cournover B, Segonds C, Favre-Bonte S 2011. Identification of Stenotrophomonas maltophilia strains isolated from environmental and clinical samples: a rapid and efficient procedure. J Appl Microbiol 111: 1185-1193.

Rademaker JLW, de Bruijin FJ 1997. Characterization and classification of microbes by REP-PCR genomic fingerprinting and computer-assisted pattern analysis. In G Caetano-Anollés, PM Gresshoff, DNA markers: protocols, applications and overviews, John Wiley and Sons, New York, p. 151-171.

Wellinghausen N, Wirths B, Franz AR, Karolyi L, Marre R, Reischl U 2004. Algorithm for the identification of bacterial pathogens in positive blood cultures by real-time LightCycler polymerase chain reaction (PCR) with sequence-specific probes. Diagn Microbiol Infect Dis 48: 229-241.

Zbinden A, Böttger EC, Bosshard PP, Zbinden R 2007. Evaluation of the colorimetric VITEK 2 card for identification of Gramnegative nonfermentative rods: comparison to $16 \mathrm{~S}$ rRNA gene sequencing. J Clin Microbiol 45: 2270-2273. 